\title{
Tendencias de la educación superior en Argentina, Chile y México*
}

\section{Trends of higher education in Argentina, Chile and Mexico}

Alejandra María Linares Cortés**

Recibido: 15 de diciembre de 2015

Revisado: 10 de marzo de 2016

Aprobado: 30 de marzo de 2016

\section{Resumen}

Este artículo expone la situación de la educación superior en Argentina, Chile y México, sus diferencias, normatividades, requisitos de ingreso, programas, enfoques, tendencias y los modelos que han desarrollado estos países para su adaptación hacia los modelos neoliberales y de globalización. Este panorama devela el inicio de la universidad en estos países latinoamericanos y da un recorrido por sus transiciones y ajustes ante nuevos

* Este estudio forma parte del estado de arte del proyecto de investigación "Educación Superior 2005- 2013 en Argentina, Colombia, Chile, España, Estados Unidos, Finlandia y México", adscrito a la Facultad de Educación de la Pontificia Universidad Javeriana de Bogotá. Cómo citar este artículo: Linares Cortés, M. A. (2016). Tendencias de la educación superior en Argentina, Chile y México. Revista CIFE, 18(28), 43-70. (DOI: http://dx.doi. org/10.15332/s0124-3551.2016.0028.02

** Licenciada en Lengua Castellana, Inglés y Francés de la Universidad de La Salle. Magistra en Educación de la Pontificia Universidad Javeriana. Correo electrónico: alejandra.linares@javeriana.edu.co 
contextos socioeconómicos y políticos. Además, pretende dar una perspectiva más amplia de la oferta académica de educación superior en estos países, que en los últimos años han atraído a estudiantes que desean ingresar a la educación superior aprovechando becas, como las ofrecidas por Argentina a los extranjeros.

Palabras clave: Educación superior, programas, enfoques, tendencias, modelos.

Clasificación JEL: I21, I22, I23, I28

\section{Abstract}

This article describes the situation of higher education in Argentina, Chile and Mexico; their differences, normativities, admission requirements, programs, approaches, trends and patterns that have developed these countries to adapt them into the neoliberal and globalization patterns. Moreover, this circumstance revealed the beginning of the university in these Latin American countries through a tour of its transitions and adjustments to new economic and political contexts. Also, it aims to give a broader perspective of the academic offer in higher education in these countries, which in recent years have attracted students who wish to join higher education taking advantage of scholarships as those offered by Argentina to foreigners.

Keywords: Higher education, programs, approaches, trends, pattern.

Classification JEL: I2 1, I22, I23, I28 


\section{Introducción}

En este artículo se presenta un panorama general de la educación superior en Argentina, Chile y México, países que en las últimas décadas han afrontado problemáticas socioeconómicas que han permeado los sistemas educativos a nivel superior. En tales sistemas se han introducido lógicas derivadas de políticas capitalistas que van en detrimento del sentido de cientificidad que le es propio a la educación y que la conducen a una función de instrucción técnica - por medio de programas de corta duración para cualificar a la mano de obra- que atienda a las necesidades del modelo económico predominante. En tal sentido, los criterios de calidad y pertinencia han sido remplazados por los de rapidez y eficacia educativa.

El presente trabajo es producto de la investigación estado del arte del proyecto de investigación "Educación Superior 2005- 2013 en Argentina, Colombia, Chile, España, Estados Unidos, Finlandia y México", adscrito a la Facultad de Educación de la Pontificia Universidad Javeriana de Bogotá. Investigación documental. Estado del arte: "La Educación Superior en Argentina, Colombia, España, Finlandia, Chile y México en los períodos 2005 a 2013”, adscrita a la Facultad de Educación de la Pontificia Universidad Javeriana de Bogotá, y cuyo fin fue develar los enfoques, las tendencias y los modelos a nivel de educación superior - los cuales coinciden con unos referentes socioeconómicos enfocados hacia la neoliberalización y la globalización-, que se encuentran permeados por las tendencias de exclusión en una sociedad que busca tener procesos de igualdad y solidaridad.

La investigación en "educación superior" contribuye especialmente a la reconstrucción teórica que se tiene de ella y dilucida conceptos para poder hablar de los procesos y aseguramientos de calidad que conllevan a brindar un mejor servicio, acorde con los actuales requerimientos socioeconómicos. Desde este contexto y con base en los principales argumentos teóricos de la educación como un bien público, este artículo presenta un diagnóstico del estado en que se encuentra actualmente la educación superior en una serie de países seleccionados; se busca confrontar las principales tendencias, la evolución y la normatividad en cada uno de los países objeto de estudio para demostrar que las preferencias de la educación muestran una relación que es proclive a aumentar la demanda de educación superior, pero sin un adecuado ajuste de la oferta con respecto a la calidad requerida.

Para mostrar esto, el documento está organizado de la siguiente manera: la primera sección corresponde a la presente introducción; en la segunda parte se realiza una breve descripción de la metodología implementada; en el tercer acápite se presenta un recuento de la educación superior de los países seleccionados, enfatizando especialmente en su normatividad, sus enfoques, sus principales tendencias y los modelos implementados; la cuarta sección expone la discusión y las conclusiones. 


\section{Aproximación metodológica}

En esta investigación documental, de corte cualitativo hermenéutico, se utilizó el "estado de arte" como instrumento para identificar los enfoques, las tendencias y los modelos en educación superior. Se retoman los rasgos ofrecidos por Escobar (2004), ya que fueron tenidos en cuenta para responder la pregunta de investigación referente a las tendencias actuales de la educación superior en varios países seleccionados de América Latina.

Se determinan ocho rasgos esenciales para la aproximación teórica, conceptual y sobre los aspectos principales de la educación y sus elementos, centrados en los siguientes puntos:

1. Fundamentado: Base teórica proveniente de revistas indexadas e investigaciones sobre el tema indagado.

2. Tematizado: Tema central y categorías, en este caso, la educación superior y sus enfoques, tendencias y modelos.

3. Acotado espacio-temporal: Período establecido de 2005 a 2013 y los países objeto del estudio(Argentina, México y Chile), que son actualmente destinos de estudios universitarios atractivos por los estudiantes de la región.

4. Sistemático: Metodología basada en análisis epistemológico y metodológico por medio del resumen analítico especializado (RAE) para realizar comparaciones.

5. Flexible: Se pueden validar nuevas categorías que surgen del proceso investigativo.

6. Crítico: Desarrollo de conceptos y teorías que emergen en el estudio.

7. Interpretativo: Surgimiento de "metaanálisis", que significa las interpretaciones que surgen del análisis, vistas de diferente manera en los países de estudio.

8. Teleológico: Sirve de apoyo a futuras investigaciones, ya que proporciona información de fuentes veraces.

Los fundamentos del estado del arte dieron a la investigación tres pasos metodológicos macro para el proceso de la información que se recaudó en los RAE (resúmenes analíticos especializados):

1. Recolección de la información por medio de la ficha RAE.

2. Fase hermenéutica: Lectura, análisis e interpretación crítica realizada en unas matrices o tablas de clasificación de la información (tabla de categorización, cuadro de análisis de regularidades discursivas y, finalmente, un compendio de resultados por países). 
3. Extracción de un balance general por medio de un diagrama de relaciones, en el que se muestran, a nivel general, los enfoques, las tendencias y los modelos de los países objeto de estudio. Posteriormente, se realizó un análisis crítico por país, el cual provee aportes de un panorama de la educación superior a nivel regional. Después, los conceptos de educación superior fueron establecidos por cada país, sintetizando su estructura y diferenciando a Argentina, Chile y México.

\section{Resultados y tendencias de la educación superior}

\subsection{Educación superior en Argentina}

El sistema de educación argentino se reguló y estructuró, en primera instancia, con la Ley Federal de Educación 24.195 de 1993 (Congreso de Argentina, 1993). Posteriormente, en 1995 se promulgó la Ley 24.521 (Congreso de Argentina, 1995), cuyo objetivo es "establecer las disposiciones de regulación, articulación, derechos y obligaciones que rigen dentro del Sistema de Educación Superior argentino" (Rojas, 2012, p. 98).

En el artículo 3.o de la Ley de Educación Superior está establecida como finalidad "proporcionar formación científica, profesional, humanística y técnica en el más alto nivel", con una conciencia ética, en la que también se hace partícipe de la preservación del medio ambiente y de una mejora de la calidad de vida que actúe en pro de la nación. Y como objetivos, en el artículo 4.o se tienen en cuenta, además de lo establecido en la Ley 24.195 (Arts. 4, 6, 19 y 22), la formación científica a nivel profesional y técnica, la diversificación de estudios a nivel superior, la promoción de la investigación y la prepararación de los docentes en todos los niveles educativos, entre otros.

Como se puede ver, los fines y objetivos responden a las actuales exigencias neoliberales que tiene la educación superior, de ahí la necesidad que se dio de actualizar la ley. El interés particular de este estudio es revisar cuáles han sido las transformaciones que ha tenido la educación superior en Argentina.

Inicialmente, su creación tuvo lugar en 1870, cuando surgieron las primeras escuelas normales; posteriormente, en 1890 aparecieron las escuelas de comercio e industria; hacia 1970, la formación de docentes de primaria fue adoptada por el SNU (sistema de educación no universitaria) para generar así el surgimiento de institutos superiores de formación docente, clasificados de la siguiente manera: independientes del Estado nacional, jurisdicción provincial y gestión privada (Rojas, 2012). La gratuidad en el sistema educativo superior tuvo su origen en 1949, bajo la presidencia de Juan Domingo Perón. 
Tabla 1. Educación superior en Argentina

\section{Educación superior en Argentina}

(Ley 24.521) título II de la educación superior ( artículos 3 al 14)

Capítulo I. De los fines y objetivos ( artículos 3 al 4 )

Requisito de ingreso: Aprobar el nivel medio o el ciclo polimodal de enseñanza.

Instituciones de educación superior pública y/o privada distribuidas así: 47 universidades nacionales; 50 universidades privadas; 7 institutos universitarios estatales; 14 institutos universitarios privados; 3 universidades provinciales; 1 universidad extranjera; 1 universidad internacional.

\begin{tabular}{|c|c|}
\hline Instituciones universitarias & Institutos terciarios \\
\hline Universidades & Formación docente. \\
\hline $\begin{array}{l}\text { Institutos universitarios (Arts. } 27 \text { y 28) Ley } \\
\text { Superior de Educación }\end{array}$ & $\begin{array}{l}\text { Formación técnico profesional (Ley de Educación } \\
\text { Técnico Profesional 26.058) (Congreso de } \\
\text { Argentina, 2005) }\end{array}$ \\
\hline $\begin{array}{l}\text { Pregrado (mínimo } 2 \text { o } 3 \text { años de duración o } 1500 \\
\text { horas) }\end{array}$ & Educación para el trabajo \\
\hline $\begin{array}{l}\text { Posgrado reglamentado por la Resolución } \\
\text { Ministerial n.o 1168/97 (especialización, } \\
\text { maestría, doctorado) (Ministerio de Cultura y } \\
\text { Educación, 1997) }\end{array}$ & \\
\hline \multicolumn{2}{|c|}{ Instituciones no universitarias } \\
\hline
\end{tabular}

Ley n.o 24.195, capítulo V (Arts. 18-24)

Formar docentes, pero no incluye a los docentes universitarios.

Ofrecen formación artística, técnico-profesional.
Colegios universitarios (Art. 22) Ley General de Educación (Decreto 1232) (Poder Ejecutivo Nacional de Argentina, 2001). Ofrecen:

a. Programas conducentes al título de técnico superior.

b. Programas de formación general o estudios básicos iguales a los que se dictan en las instituciones universitarias, que estarán a cargo de los docentes de los colegios universitarios que posean título Universitario.

c. Programas de formación de docentes para todos los niveles de la educación, excepto el nivel superior.

d. Programas cortos, flexibles y/o a término, que faciliten la capacitación, el perfeccionamiento, la reconversión de competencias técnicas y profesionales que tiendan a hacer posible la inserción laboral del egresado. 
Actualmente, Argentina es uno de los países latinoamericanos que cuenta con una tasa alta de cobertura en educación. En este sistema también hay falencias, como la oferta de matrícula en carreras universitarias tales como Derecho, Economía y Administración. En los institutos de educación terciaria; la administración informática y de paramédicos, no concuerdan con la demanda laboral existente (Rojas, 2012, pp.112-113). De esta manera, sería muy conveniente que existiera una mejor articulación en la educación superior ( $\mathrm{SU}$ ) y el sistema de educación no universitaria (SNU) con las necesidades del contexto laboral. En el análisis se puede ver cuál es la oferta que tienen las instituciones universitarias, las no universitarias y los institutos terciarios; en Argentina, estos últimos se refieren a las instituciones de educación técnica y a los SNU de corta duración (dos o tres años).

Las modalidades de estudio ofrecidas son presencial y a distancia, con programas muy heterogéneos que cuentan con apoyos de recursos tecnológicos (TIC). Por lo general, los programas de posgrado - como especializaciones, maestrías y doctorados- pueden ser lo que en Argentina se llama "arancelados", es decir, las tarifas dependen de cada institución. De igual manera, existen estudios posgraduales que cuentan con sistemas de becas para estudiantes extranjeros. La educación superior en Argentina se da en instituciones universitarias, instituciones no universitarias y en los institutos terciarios. En la tabla 1 se pueden ver sus requisitos de ingreso, su clasificación, las instituciones y el tipo de programas oferentes.

\subsection{Enfoques de educación superior en Argentina}

Los enfoques hallados en este rastreo documental en Argentina de 2005 a 2013 responden a los últimos cambios que ha tenido la educación en este país en los últimos tiempos. Básicamente son cuatro, los cuales se explicarán a continuación:

\section{Interculturalidad}

Argentina es un país que socialmente cuenta con población afrodescendiente e indígena, por esta razón, instituciones como la UNESCO- IESALC se interesan por estos grupos sociales y hacen estudios en los que reflejan su situación en cuanto a educación superior se refiere. Los enfoques hallados (Millán, 2012) permiten decir que la actual Ley de Educación Superior 24.521 de 1995 (Congreso de Argentina, 1995) sí tiene en cuenta la educación superior dirigida hacia estos grupos sociales por medio de la educación superior intercultural. Ejemplo de esto se menciona en el segundo artículo de la Ley 24.521 de Educación.

En cuanto a los pueblos indígenas u originarios de la región, en la Ley 24.521 de 1995 existen unos lineamientos que se refieren específicamente a cada una de las regiones con esta población, discriminados de la siguiente manera y citados por (Millán, 2012): 
Capítulo IV, artículo 75, inciso 17, en el que se responsabiliza al Congreso del reconocimiento étnico y cultural de la población indígena de Argentina y de ofrecer también el derecho a una educación "bilingüe e intercultural”, estipulada en la Ley 26.206 del 27 de diciembre de 2006 (Congreso de Argentina, 2006), capítulo XI, artículo 52, a nivel de primaria y secundaria.

A partir de la inclusión que se hace de los pueblos originarios en la nueva Ley de Educación 24.521 de 1995 es que cuentan con apoyo de programas de becas como las que otorga el INAI (Instituto de Asuntos Indígenas) de la Ley n.o 23.302 (Congreso de Argentina, 1985). Lastimosamente, este beneficio no se visualiza para la población afrodescendiente, pero lo que sí se refleja es el trabajo de entidades como CONAFRO (Consejo Nacional de Organizaciones Afros de la Argentina) y DIAFAR (Asociación Civil África y su Diáspora Africana de la Argentina), las cuales trabajan en pro de las políticas públicas concernientes a esta población.

\section{Globalización}

El segundo enfoque es la globalización, que permea continuamente a la educación en general. Esta se vincula por medio de las TIC, las cuales se han convertido en un apoyo y le han dado una perspectiva bimodal, la cual, según Santángelo (2011), plantea que:

La $\mathrm{EaD}$ va camino a transformarse en Enseñanzas Mediadas por Tecnologías de la Información y la Comunicación (EMTIC), dentro de la amplia oferta universitaria, de cara a las transformaciones socioculturales y su posible impacto en esas instituciones por el efecto de los procesos de digitalización - globalización y fragmentación- y sus consecuencias. (p. 22).

Del mismo modo, la EaD (educación a distancia) de Argentina debe cumplir con requisitos similares a la educación presencial, además, ha de tener en cuenta el área de influencia a la cual dará cobertura; las influencias tecnológicas, científicas y socioeconómicas; su pertinencia; el perfil del alumno y la evaluación, entre otros aspectos. En el Decreto Reglamentario n.o 81 del 22 de enero de 1998 (Poder Ejecutivo Nacional de Argentina, 1998) y en la Resolución Ministerial n.o 1717 del 29 de diciembre de 2004 (Ministerio de Educación, Ciencia y Tecnología, 2004) se pueden consultar ampliamente estas disposiciones (Santángelo, 2011).

\section{Neoliberalismo}

Como consecuencia del golpe de estado de 1976, el tercer enfoque es el neoliberalismo, al cual se refiere Foucault (citado por Martínez, 2010) cuando dice que en él surge la teoría del Homo oeconomicus, que resulta ser vista como "subjetividad" pensada como "empresario de sí mismo" y que da la base al "capital humano", que saldrá como producto de las nuevas reformas educativas establecidas, para que responda al modelo del 
mercado que se necesita en el contexto. Por esta razón, hacia el año 1990 se acrecentó el mercado universitario privado con una demanda heterogénea de carreras en el SU (sistema de educación superior) y en el SNU (sistema de educación no universitaria) (Rojas, 2012), para reproducir sujetos que puedan responder con calidad en el sector productivo, el cual atiende a políticas socioeconómicas. Así, por medio de una expansión de programas a nivel técnico, se permitió que en poco tiempo (dos o tres años) hubiera una mayor cualificación de la mano de obra requerida para cumplir con las solicitudes de aperturas comerciales que se dieron en los inicios del neoliberalismo.

\section{Reconocimiento}

El cuarto enfoque es el de "reconocimiento", que surge desde la educación superior pública como consecuencia de supervivencia ante los efectos de la neoliberalización y la globalización, que la llevan a formar a los sujetos para el trabajo y para así responder a estándares internacionales, pero que también la dirigen hacia una calidad educativa mediada por mecanismos como la acreditación de sus procesos. Mollis (2008) menciona los siguientes parámetros: "Reconocimiento a déficit pedagógico, mala representatividad docente en lo administrativo, proyección de la investigación contextualizada, promoción de líderes políticos y reflexión del sentido, misión y praxis de la universidad” (p. 530).

En lo relacionado con el "déficit pedagógico", se sugiere tener en cuenta una mejor formación de docentes para que estos posean una cualificación adecuada a la educación superior. La "mala representatividad docente en lo administrativo" se refiere a que debe haber meritocracia en estos cargos para que así los docentes que estén vinculados a lo administrativo realmente trabajen en pro de la comunidad universitaria. Por su parte, la "proyección de la investigación contextualizada" corresponde a que se debe tener más en cuenta el contexto social para actuar en pro de del país. En lo que respecta a la "promoción de líderes políticos", la tarea es formar sujetos con sentido político y ético que luchen por la justicia y la equidad. Finalmente, la "reflexión del sentido, misión y praxis de la universidad" se enfoca hacia el sentido inicial de ciencia que debiera tener la educación superior, no a los enfoques y direccionamiento que ha alcanzado en los últimos tiempos. Desde esta perspectiva, tales parámetros de reconocimiento son fundamentales para recobrar el enfoque académico inicial que tenía la educación superior pública, la cual era más reflexiva y con sentido científico, en vez de uno neoliberal como el actual.

Para concluir, con base en el rastreo documental se puede decir que los enfoques hallados de la educación superior argentina la dirigen al cumplimiento de los requerimientos que determinan entidades internacionales para adecuarse a la realidad socioeconómica que está viviendo en general América Latina. Se inicia con enfoques que deben responder a la cobertura de la educación superior que han de tener los pueblos originarios y la población afrodescendiente, ya que son poblaciones que han tomado fuerza en el respeto de sus derechos en los últimos treinta años. Posteriormente, es un hecho que los 
enfoques deben responder a los requerimientos que se hacen desde la neoliberalización y la globalización, y que le dan a la educación superior la necesidad de actualizarse y dar una cobertura a nivel de educación a distancia por medio de recursos como las TIC. De igual manera, no se puede olvidar el sentido inicial de la educación superior, y es por eso que Mollis (2008) hace un llamado por medio del enfoque de "reconocimiento".

\subsection{Tendencias en educación superior en Argentina}

Las tendencias halladas en el rastreo documental de la educación superior en Argentina 2005-2013 son las siguientes: cohesión social, normatividad en educación superior en el programa de EaD, expansión del SNU (sistema de educación no universitaria), políticas de evaluación y acreditación, preferencia por la universidad pública. Todas estas tendencias responden al modelo neoliberal que se ha presentado desde los años noventa y también a lo planteado en la Ley Nacional de Educación 24.521 de 1995. A continuación se enuncian y explican tales tendencias:

- Tendencia de cohesión social (Al Gepal, s. f. p. 13): En ella se refleja la inclusión que hay no solo de la población indígena, sino también de los afroargentinos. (Millán, 2012) afirma que "en la actualidad, los afroargentinos se han conformado en instituciones que reivindican sus derechos, haciéndose cada día más visibles". (p. 104). La pertenencia a grupos organismos como CONAFRO (Consejo Nacional de Organizaciones Afros de la Argentina) y DIAFAR (la Diáspora Africana de la Argentina) hacen que en el país, esta población se sienta respaldada en la reivindicación de sus derechos. La educación superior para esta población es una forma de compensación ante la sociedad después del maltrato recibido en la época de la Colonia por medio de la esclavitud.

- Tendencia de Normatividad de la Educación a Distancia: Se ve encaminada hacia la reglamentación para que haya mayor credibilidad y para mejorar la formación de los docentes que imparten este tipo de educación. En muchos casos es una buena opción que se toma para continuar con los estudios en educación superior, ya que puede llegar a todas las partes de la región. Así, Santángelo (2011) afirma que "es muy necesario analizar y comprender las limitaciones y posibilidades que constituyen el diseño de plataformas digitales como entornos innovadores de aprendizaje (p. 22), debido a que estas herramientas tecnológicas permiten que se fortalezcan los programas y haya una comunicación más directa con la clase y el profesor o tutor virtual.

- Tendencia de la expansión del SNU (sistema de educación no universitaria): Se ofrece mediante los programas de carreras técnicas; se ha convertido en una de las tendencias más relevantes de la educación superior argentina debido a que su titulación se hace más rápida y responde a las necesidades del sector 
productivo. Rojas (2012) propone una mejor articulación en la educación de nivel medio para que el estudiante pueda hacer un "nivel transversal" o "ciclo básico" en los institutos terciarios, con el fin de brindar al estudiante la opción de continuar con sus estudios universitarios o, si lo prefiere, para que incursione en la vida laboral. Este es el tipo de reestructuración que hace falta, pues muchas veces al salir de la educación secundaria a la terciaria no existe una real convicción de la carrera que se quiere hacer. Otra de las tendencias que se da a raíz de esta falta de reestructuración es que al querer pasar de la formación SNU a la SU, a veces no hay espacio para la homologación de títulos.

- Tendencia de políticas de evaluación y acreditación: Se refiere al proceso que tienen las IES y las carreras universitarias, incluidos los posgrados. Esto ha sido declarado como "política de promoción de calidad" a partir de la creación de la Secretaría de Políticas Universitarias en 1994 y la Ley de Educación Superior 24.521 de 1995 (Mollis, 2008). De allí surgen la CAP (Comisión Nacional de Posgrados) y la CONEAU (Comisión Nacional de Evaluación y Acreditación Universitaria), cuya misión es supervisar estos procesos. De esta manera, se cumplen los requerimientos dados por el neoliberalismo y la globalización en la educación.

- En cuanto a la tendencia por la preferencia por la universidad pública, esta se da obviamente por su gratuidad. Al respecto, Dirie, Mollis y otros (2001), citados por Mollis (2008) opinan que:

Como consecuencia, el nivel superior de educación ofrece una variedad de instituciones que difieren en la calidad y en sus misiones específicas. Así se desarrolló una fragmentación costosa y conspirativa contra el funcionamiento eficiente del conjunto del nivel superior de educación debido a que los sectores que lo conforman nunca establecieron canales de articulación entre los distintos tipos de formación que el nivel superior brindaba (universitaria y no universitaria) y nunca se reciclaron las ofertas educativas ya instaladas. (Mollis, 2008, p. 514).

$\mathrm{Al}$ respecto, se puede decir que esa variedad lo que produce es una educación superior sin articulación entre la educación universitaria y la no universitaria, lo cual ocasiona problemas de homologación en caso de que el estudiante quiera seguir en su profesionalización. En Argentina, las universidades cumplen con roles de multifuncionalidad y heterogeneidad; esto significa que simultáneamente pueden vender servicios educativos, entrenar al estudiante en una disciplina, entrenar a los profesionales e investigar, entre otras funciones (Mollis, 2008).

\subsection{Modelos de educación superior en Argentina}

Los modelos hallados en el rastreo documental de educación superior en Argentina de 2005 a 2013 responden a las necesidades que tiene el país para estar a la vanguardia de 
las necesidades del contexto social, el cual exige una formación acorde con las exigencias del sistema productivo y global. Esta es una de las razones por las cuales se tuvo que pensar en una ley de educación pertinente a estas necesidades, y como resultado surgió la Ley 24.521 de 1995, en la que los niveles SU (superior universitario) y SNU (superior no universitario) cuentan con normatividades específicas cuya puesta en común es responder a las necesidades políticas. Justamente, Mollis (2007, p. 73, citada por Rojas, 2012) dice que los niveles SU (superior universitario) y SNU (superior no universitario) poseen políticas educativas que responden a intereses propios de los gobiernos y modelos económicos. Esta puede ser una de las razones por las cuales el modelo de educación superior es heterogéneo y diversificado. Dentro de estas características, el SNU da soluciones para cubrir las necesidades del sector productivo por medio de un modelo de formación laboral que ofrece gran variedad de carreras técnicas de corta duración, brindando así capacitación acorde con las necesidades del sector productivo, el cual presenta tasas altas de desempleo.

Otra de las reestructuraciones que se dieron en la nueva Ley de Educación de Argentina fue pensar en un modelo de interculturalidad e inclusión (Millán, 2012), ya que se tienen en cuenta a las comunidades indígenas y afrodescendientes que pertenecen a la región, de manera que se promueve así un fortalecimiento cultural y el acceso a la educación superior. En cuanto a la educación superior a distancia, se sugiere un modelo educativo de referencia. Santángelo (2011) menciona que "este componente está considerado de importancia central, ya que desde él mismo es que deben definirse - de manera coherente- las características y funcionalidades, evitando fragmentaciones en la estructura formativa general" (p. 20). Debido a que la educación a distancia debe tener similitudes con la presencial, este modelo se hace fundamental porque desde este se plantean las bases pedagógicas, epistemológicas y metodológicas.

Por último, el modelo mercantilista - el cual no solamente lo posee Argentina, sino también la mayoría de los países latinoamericanos- se debe a que la educación superior de esta parte del continente se dejó permear de la globalización y la neoliberalización, por ende, le correspondió como misión la parte de la cualificación de la mano de obra y no tanto la preocupación por el desarrollo científico y tecnológico (Mollis, 2008). No hay que olvidar que Argentina es uno de los países latinoamericanos que menos invierte en educación, por eso la demanda de programas de nivel SNU (superior no universitario) y a nivel privado se acrecentó tanto. Lastimosamente, el modelo mercantilista llegó para quedarse dentro de las instituciones educativas, provocando así variedad de oferta que solo está comprometida con los modelos económicos y políticos.

\subsection{Educación superior en Chile}

Inicialmente, la educación superior fue asumida por religiosos, siendo los dominicos los encargados de fundar la primera universidad en 1962: Universidad de Santo 
Tomás; posteriormente llegaron los jesuitas y finalmente fue hasta 1842 que surgió la Universidad de Chile, de carácter estatal. Bernasconi y Contreras (2004) aluden a las cuatro etapas que asumió la educación superior antes de la reforma universitaria de 1967-1968:

a) El período anterior a la reforma universitaria de 1967-68; b) la era de la reforma, que termina con el golpe de Estado de 1973; c) el período de la intervención militar anterior a la reforma de 1980; y d) la etapa que comienza con las reformas de 1981 y que perdura hasta hoy. (p. 17).

La etapa (d) que se menciona se refiere a las tendencias neoliberalistas y privatistas (Catani, Campbell y De Sousa, 2005) que se dieron en este período y que le dieron un nuevo giro al sistema superior con la formación de institutos profesionales (IP) y centros de formación técnica (CFT).

Actualmente, la educación superior en Chile está compuesta por tres tipos de instituciones: universidades, IP (institutos profesionales) y CFT (centros de formación técnica), todos regidos por la Ley Orgánica Constitucional de Enseñanza (LOCE n.o 18.962) del Ministerio de Educación, publicada el 10 de marzo de 1990, que actúa como marco de referencia. En la tabla 2 se describe la conformación, el requisito de ingreso, el tipo de institución y las entidades que brindan los diferentes programas.

Tabla 2. Educación superior en Chile

\section{Educación superior en Chile}

Ley Orgánica Constitucional de Enseñanza (n.o 18.962) del Ministerio de Educación, publicada el 10 de marzo de 1990.

Requisito de ingreso:

Cada institución lo define.

Presentar la licencia de enseñanza media chilena o del exterior, la cual será aprobada por el Ministerio de Educación.

Tipo de institución

Universidad

Estatales (16)

Particulares con aportes del Estado (9), creadas antes de 1980 .

\section{Descripción}

\section{Carreras profesionales}

Duración de 8 a 10 semestres (4 o 5 años)

Carreras técnicas de nivel superior

Duración: de 4 a 6 semestres (2 y 3 años)

Privadas (35), creadas después de 1980 a partir de lo establecido en el DFL 1 de 1980 o la Ley 18.962 de 1990. 


\section{Educación superior en Chile}

IP (institutos

profesionales)

Imparten carreras profesionales y técnicas de nivel superior, como las universidades, pero, a diferencia de ellas, no pueden entregar grados académicos.

La mayoría de las carreras profesionales dura 8 semestres (4 años).

Existe un conjunto de títulos profesionales definidos por ley que requieren haber obtenido previamente el grado académico de licenciado, por lo que en esos casos solo pueden ser otorgados por una universidad.

Esos títulos son:

Abogado (licenciado en Ciencias Jurídicas); arquitecto (licenciado en Arquitectura); bioquímico (licenciado en Bioquímica); cirujano dentista (licenciado en Odontología); ingeniero agrónomo (licenciado en Agronomía); ingeniero civil (licenciado en Ciencias de la Ingeniería); ingeniero comercial (licenciado en Ciencias Económicas o licenciado en Ciencias en la Administración de Empresas); ingeniero forestal (licenciado en Ingeniería Forestal); médico cirujano (licenciado en Medicina); médico veterinario (licenciado en Medicina Veterinaria); psicólogo (licenciado en Psicología); químico farmacéutico (licenciado en Farmacia); profesor de Educación Básica (licenciado en Educación).

Carreras profesionales: Son las que entregan una formación general y científica para un adecuado desempeño profesional y que pueden obtenerse en la universidad o en un IP. Sin embargo, existe un conjunto de títulos profesionales definidos por ley que requieren haber obtenido previamente el grado académico de licenciado, por lo que en esos casos solo pueden ser otorgados por una universidad.

Carreras técnicas: Se trata de carreras que confieren la capacidad y los conocimientos necesarios para desempeñarse en una especialidad de apoyo al nivel profesional, o bien, desempeñarse por cuenta propia. Para obtener el título de técnico de nivel superior es necesario aprobar un programa de estudios de una duración mínima de 1600 horas de clases (4 semestres).

\section{CFT (centros de} formación técnica)

Carreras técnicas de nivel superior.

Orientadas a entregar a los estudiantes la capacidad y los conocimientos necesarios para desempeñarse en una especialidad de apoyo al nivel profesional, o bien, desempeñarse por cuenta propia.

Para optar al título de técnico de nivel superior es necesario aprobar un programa de estudios de una duración mínima de 1600 horas de clases (4 semestres)

La mayoría de las carreras técnicas duran entre 4 semestres ( 2 años) y 6 semestres (3 años).

\section{Educación a distancia}

En Chile hay universidades a distancia que ofrecen carreras en línea y cursos virtuales.

Fuente: elaboración propia. 


\subsection{Enfoques de educación superior en Chile}

Los enfoques hallados en el rastreo documental de la educación superior en Chile 20052013 se dirigen hacia su privatización. Esto se remonta a inicios del siglo XX con el surgimiento de las universidades católicas (Méndez, Campbell y De Sousa, 2005) y su mercadización, que se da precisamente porque debe responder a un modelo neoliberal económico (Brunner y Uribe, 2007). Estos perfiles hacen que la universidad no solo se piense desde lo académico, sino que también entre en una competencia mercantilista que ofrezca un servicio que cumple con estándares de calidad a sus "clientes". Esto es fortalecido por medio del ofrecimiento de nuevos programas, tecnología, instalaciones, planta cualificada de docentes, todo ello para poder obtener mejores fuentes de ingresos para su autosostenimiento.

Otro enfoque es el de la virtualización de la educación superior a distancia, que ayuda a que los procesos de mercadización se cumplan, pues los programas a distancia logran llegar hasta las regiones más apartadas, contando ahora con el refuerzo y la complementariedad de la virtualidad, quedando así unos programas semipresenciales con enfoque virtual (Farcas, 2010). Por otra parte, en Chile no hay enfoques dirigidos a la población indígena y afrodescendiente, no hay políticas públicas definidas al respecto. La población indígena puede acceder a programas de formación docente dirigida a jóvenes pertenecientes a pueblos originarios, esto es a manera de promoción y resaltando la importancia de esta población. Lastimosamente, con los afrodescendientes no ha habido visibilización (Mora, 2012).

De esta manera, queda visto que es indispensable replantear en Chile la educación intercultural, ya que solo con el enfoque de formación a jóvenes de ascendencia indígena no se solucionan las problemáticas de inclusión y racismo para poder hablar de pluriculturalidad.

\subsection{Tendencias de educación superior en Chile}

Las tendencias halladas en el rastreo documental de la educación superior en Chile 2005-2013 son: privatización, cualificación de la mano de obra, educación a distancia y virtual (B-Learning; E-learning; Life long learning; E-support) y transfronterizacion de la educación superior. Estas tendencias surgieron como respuesta a los modelos neoliberales y de globalización que se iniciaron en los años ochenta en Chile y se ajustaron así al nuevo perfil de universidad.

\section{Primera tendencia: de privatización}

Se dio en las décadas de los ochenta y los noventa, con 31 universidades (Vejar, 2013). Surgió durante el Gobierno de Pinochet, el cual cambió el sentido de promoción social que se tenía en la universidad a comienzos de los años setenta y dio paso así a un acceso 
más amplio a la educación superior por parte de las clases sociales media y alta, pero dejó relegada a la clase baja (Méndez, Campbell y De Sousa, 2005). Fue a partir de la reforma de 1981 que se presentó la expansión del sector privado. Bernasconi y Rojas (citados por Vejar, 2013) manifiestan que "se diversificaron las instituciones de educación superior en institutos profesionales y centros de formación especializada" (p. 73), lo cual generó nuevos programas, incluyendo el nivel de especialización.

\section{Segunda tendencia: cualificación de la mano de obra}

Se da debido a la gran demanda de instituciones educativas de nivel técnico de fácil accesibilidad por sus tarifas y tiempo, ya que ofrecen programas de corta duración para la cualificación en un oficio. Lamentablemente, esto ha conllevado una sobrecualificación de la mano de obra (Weller, citado por Vejar, 2013). De esta manera, se genera poca concordancia con lo que realmente necesitan el mercado y la formación que están dando las instituciones. Esto crea al mismo tiempo una desvalorización de la mano de obra y origina el subempleo y el ingreso al sector productivo devengando salarios bajos no justificables con la formación académica.

\section{Tercera tendencia: educación a distancia y virtual}

\section{(B- Learning; E- learning; Life long learning; E-support)}

Esta tendencia ha tenido más desempeño en la educación no formal con cursos de corta duración y diplomados (Santander, Schalk, Zavando y Durán, 2011). Estos programas han sido apoyados por el uso de las TIC. Sus programas tienen tres variaciones: B-learning, referida a la universidad semipresencial; E- learning, que brinda la posibilidad de estudiar virtualmente desde cualquier sitio sin necesidad de asistir a una institución educativa; un ejemplo de este sistema es la UNIACG (Universidad de Artes, Ciencias y Comunicación); Life long learning, que constituye todo el background o el conocimiento previo que tiene una persona y lo vincula con el E-learning; finalmente está el $E$-support, que se refiere al soporte que la virtualidad le da a la educación presencial (Farcas, 2010).

\section{Cuarta tendencia: educación transfronteriza}

Se puede dar por medio del uso del E-learning y también mediante convenios que las universidades chilenas públicas o privadas establecen con otros países para que sus estudiantes y/o docentes viajen en calidad de intercambios estudiantiles.

\subsection{Modelos de educación superior en Chile}

Los modelos hallados en el rastreo documental de educación superior en Chile de 2005 a 2013 se enfocan básicamente en la reestructuración que se dio a la educación superior 
a principios de los años ochenta debido a las transformaciones socioeconómicas que tuvieron lugar en este país. Inicialmente, los modelos de la universidad eran el burócrata (trabajo-docencia-investigación) y el humboldtiano (servicio en pro del saber) (Brunner y Uribe, 2007). Estos modelos tomaron otro rumbo debido a las transformaciones sociopolíticas, a la neoliberalización y a la globalización, entre otros. Así surgió el modelo de universidad neoliberal, que es muy similar al perfil de universidad que le da Martínez (2010), en el cual la plantea como "productora de productores", donde "el sujeto formado en la universidad debe ser tratado con la misma disposición que la que garantiza la calidad de los procesos de certificación de un producto cualquiera" (p. 155). En la actualidad, este modelo es inherente a la misión de las universidades, ya que se piensa al estudiante como un producto de calidad que el mercado necesita.

Para la adaptación de esta nueva misión, las reformas de los años ochenta, mediante modelos de gestión y reestructuración de la educación superior, permitieron la creación de nuevas instituciones públicas a partir de sedes universitarias regionales, instituciones privadas, institutos profesionales (IP) y centros de formación técnica (CFT) (Vejar, 2013), los cuales brindan formación técnica y tecnológica en menos tiempo y acordes con las necesidades del mercado. Seguidamente, una estrategia de cobertura y capacitación en educación superior la conforma el modelo de la educación a distancia virtual, que es una oportunidad de acceso a ella por varios sectores socioeconómicos del país, por ejemplo, quienes cuentan con dificultades de tiempo para asistir a una institución, como en el caso de los mineros, o de distancia, que puede ser un problema para el campesinado del país, entre otros aspectos.

Por su parte, Santander, Schalk, Zavando y Durán (2011) mencionan que "en Chile, la educación superior a distancia no cuenta con normas específicas que regulen esta modalidad. No obstante, se ha ido desarrollando una oferta variada, incentivada principalmente por la masificación de las tecnologías de la información e Internet” (pp. 6768). Esta falta de normatividad es la que hace que, a pesar de su cobertura y acceso, la virtualidad no tenga una buena acogida por parte de algunos sectores.

\subsection{Educación superior en México}

En México, la educación superior no cuenta con una ley específica, lo único que se encuentra es el artículo 37 de la Ley General de Educación (Congreso de los Estados Unidos Mexicanos, 1993) -en el que se menciona su clasificación- y la Ley para la Coordinación de la Educación Superior, de diciembre 29 de 1978 (Congreso de los Estados Unidos Mexicanos, 1978), cuyo objeto es que se cumpla en todo el Estado y que haya una coordinación de la educación superior; esta coordinación tiene un rol de veeduría para que se cumplan las funciones que esta misma estipula. Por otro lado, las políticas públicas han permitido un crecimiento de la educación superior a nivel privado (De Garay, 2013). Tal crecimiento se ha venido dando desde los años ochenta, cuando 
la mayoría de los países latinoamericanos, sin excluir a México, vieron envueltos sus sistemas de educación superior en modelos neoliberales en los que se les descargaba la responsabilidad de cualificar la mano de obra a nivel de la educación terciaria y a nivel técnico, para así responder a las demandas del mercado.

La educación superior en el país azteca puede ser pública, privada, federal o estatal, y presencial o a distancia. Sus niveles son técnico superior universitario y profesional asociado; licenciatura, que incluye la educación normal; posgrado (especialidad, maestría, doctorado) (para mayor información, ver tabla 3). La educación pública cuenta con diferentes tipos de instituciones, como el SNIT (Sistema Nacional de Institutos Tecnológicos), universidades públicas federales y estatales, universidades públicas estatales con apoyo solidario, universidades politécnicas y tecnológicas, universidades interculturales, centros públicos de investigación, escuelas normales públicas y otras instituciones públicas (ver tabla 4).

\section{Tabla 3. Educación superior en México}

\section{Educación superior en México}

Art. 37. Ley General de Educación. (El tipo superior es el que se imparte después del bachillerato o de sus equivalentes. Está compuesto por la licenciatura, la especialidad, la maestría y el doctorado, así como por opciones terminales previas a la conclusión de la licenciatura. Comprende la educación normal en todos sus niveles y especialidades).

Requisito de ingreso: Establecido por cada institución

Pública y/o privada - Federal/ Estatal

\section{Nivel}

Técnico superior universitario y profesional asociado

Entidades: universidades tecnológicas, otros.

Licenciatura, que incluye la educación normal.

\section{Descripción}

Requiere estudios de bachillerato, forma profesionistas técnicamente capacitados para el trabajo en una disciplina específica, sus programas de estudio son de dos años y se tiene la posibilidad de estudiar posteriormente dos años más y lograr una licenciatura o una ingeniería.

Es de carácter terminal y forma profesionistas en las diversas áreas del conocimiento con programas de estudio de cuatro años o más.

Entidades: instituciones

tecnológicas, universidades.

Formacion de maestros (normal)

Posgrado (universidades):

Requiere la licenciatura y forma profesionistas con alto grado de especialización profesional, que se acreditan mediante un título o grado.

Especialidad, maestría, doctorado. 
Educación a distancia

Sistema en el que el estudiante puede combinar estudio y trabajo. El plan de estudio se desarrolla haciendo uso de TIC.
La UNAM, el IPN e incluso la SEP ya cuentan con programas de educación superior abierta y a distancia en los cuales los alumnos pueden estudiar en línea las carreras de Ciencias de la Comunicación, Ciencias Políticas y Administración Pública, Contaduría, Derecho, Economía, Relaciones Internacionales, Sociología, Trabajo Social, Bibliotecología y Pedagogía, entre otras.

Fuente: elaboración propia

Tabla 4. Instituciones de educación superior públicas en México

\section{Instituciones de educación superior públicas en México}

\section{SNIT (Sistema}

Nacional de Institutos

Tecnológicos)

Integrado por 218

instituciones en los 31

estados de la república mexicana.

federales

Universidades públicas estatales

Universidades públicas estatales con apoyo solidario

Universidades tecnológicas (UT)
Formación de profesionales competentes mediante planes y programas de estudios pertinentes a cada región.

Los institutos tecnológicos (ITES) se encargan de:

-Formación de ingenieros.

-Formación de profesionales de las áreas administrativas.

Se distribuyen en:

-Instituto Tecnológicos Federales

-Institutos Tecnológicos Estatales

Cumplen con funciones de docencia e investigación (generación y aplicación innovadora del conocimiento), y de extensión y difusión de la cultura.

IES creadas por decreto de los congresos locales, bajo la figura jurídica de organismos públicos descentralizados. Estas instituciones estatales desarrollan las funciones de docencia, generación y aplicación innovadora del conocimiento, así como de extensión y difusión de la cultura.

Reciben aportaciones del programa presupuestario. Su financiamiento proviene principalmente de los gobiernos estatales; así mismo, el Gobierno federal contribuye con un apoyo solidario convenido con el estado respectivo. Al igual que las universidades públicas estatales, desarrollan las funciones de docencia, generación y aplicación innovadora del conocimiento, así como de extensión y difusión de la cultura.

Ofrecen a los estudiantes que terminan la educación media superior una formación intensiva que les permite incorporarse en corto tiempo (luego de dos años) al trabajo productivo o continuar estudios a nivel de licenciatura en otras instituciones de educación superior. El modelo educativo de las UT está orientado al aprendizaje como un proceso a lo largo de la vida, enfocado en el análisis, la interpretación y el buen uso de la información. Actualmente hay 61 universidades tecnológicas en 26 estados de la república. Quien estudia en estas instituciones obtiene el título de técnico superior universitario. 
Instituciones de educación superior públicas en México

$\begin{array}{ll} & \text { Son un proyecto educativo creado en } 2001 \text { para ofrecer carreras de } \\ & \text { ingeniería, licenciatura y estudios de posgrado al nivel de especialidad. } \\ \text { Universidades } & \text { Sus programas, son diseñados con base en el modelo educativo basado } \\ \text { politécnicas } & \text { en competencias y se orientan en la investigación aplicada al desarrollo } \\ & \text { tecnológico; al mismo tiempo, llevan una colaboración estrecha con } \\ & \text { organizaciones de los sectores productivo, público y social. }\end{array}$

Misión: Promover la formación de profesionales comprometidos con el desarrollo económico, social y cultural, particularmente de los pueblos indígenas del país y del mundo circundante; revalorar los conocimientos de los pueblos indígenas y propiciar un proceso de síntesis con los avances del conocimiento científico; fomentar la difusión de los valores propios de las comunidades, así como abrir espacios para promover la revitalización, el

Universidades desarrollo y la consolidación de lenguas y culturas originarias.

interculturales

Objetivos: Impartir programas formativos en los niveles de profesional asociado, licenciatura, especialización, maestría y doctorado, pertinentes al desarrollo regional, estatal y nacional, orientados a formar profesionales comprometidos con el desarrollo económico, social y cultural en los ámbitos comunitario, regional y nacional, cuyas actividades contribuyan a promover un proceso de valoración y revitalización de las lenguas y las culturas originarias.

Conformados por centros públicos de investigación CONACYT, centros de investigación del IPN, así como de los estados de Tamaulipas, Jalisco y Chihuahua, respectivamente, y de la UNAM. Tienen entre sus objetivos

Centros públicos de investigación principales los siguientes: divulgar en la sociedad la ciencia y tecnología; innovar en la generación, el desarrollo, la asimilación y aplicación del conocimiento de ciencia y tecnología; vincular la ciencia y la tecnología en la sociedad y el sector productivo para atender problemas; y crear y desarrollar mecanismos e incentivos que propicien la contribución del sector privado en el desarrollo científico y tecnológico.

Forma profesores de educación preescolar, primaria y secundaria, labor

Escuelas normales públicas que realizan por medio de la red de normales a nivel nacional. Las escuelas de educación normal superior ofrecen, entre otros, programas de licenciatura en Educación Preescolar, Primaria, Primaria Intercultural Bilingüe, Secundaria, Especial, Inicial, Física y Artística.

El sistema de educación superior pública en México es diverso, por lo Otras instituciones públicas tanto, existen instituciones que, debido a sus características particulares, no pueden ser ubicadas dentro de alguno de los subsistemas anteriores.

Fuente: elaboración propia.

\subsection{Enfoques de educación superior en México}

Los enfoques hallados en este rastreo documental desde el año 2005 hasta 2013 responden a los cambios que ha tenido la educación superior mexicana en sus últimas décadas. La mayoría de ellos apuntan hacia la universidad intercultural, la inclusión, la diversidad cultural, la cohesión social y políticas de equidad. Todos estos enfoques surgen 
debido a que en el país existe variedad de pueblos indígenas, los cuales son tenidos en cuenta en el Marco normativo en materia de equidad y género -de educación- Art. 3 (Alcántara y Navarrete, 2014).

Por consiguiente, es indudable que, en México, la normatividad actúa en pro de una igualdad de acceso a la educación en todos sus niveles, incluyendo la media superior. Incluso organismos como la OIT (Organización Internacional del Trabajo) y la UNESCO (Organización de las Naciones Unidas para la Educación la Ciencia y la Cultura) se han unido para trabajar en pro de los pueblos originarios y afrodescendientes, pero, lamentablemente, las leyes mexicanas no están enfocadas hacia estos últimos. La implementación de universidades interculturales fue pensada para favorecer a los pueblos originarios; al respecto, Bastida (2012) menciona: "Estas universidades tienen un modelo propio sustentado en los principios de la interculturalidad y, además, sus licenciaturas están pensadas para resolver problemas locales y fortalecer procesos identitarios que muchas poblaciones han perdido" (p. 353).

Sin embargo, a pesar de que la ley de los Estados Unidos Mexicanos ha estado muy pendiente en cuanto a los temas de inclusión, discriminación y cohesión social, aún se presenta una baja demanda de asistencia a la educación superior por parte de los pueblos identitarios. Una de las razones puede ser la que se argumenta con base en la experiencia que se dio en la UIEM (Universidad Intercultural del Estado de México), según la cual factores como la distancia de los centros educativos y la poca pertinencia de los programas ofrecidos no eran adecuados a sus contextos. Los bajos recursos económicos y una buena formación académica secundaria que los prepare para que den rendimiento en la educación superior hacen que no haya interés por parte de algunos miembros de los pueblos originarios (Bastida, 2012).

Otro enfoque que emerge en la educación superior en México es la educación a distancia, la cual cuenta con apoyo del Instituto Latinoamericano de la Comunicación Educativa (ILCE) como entidad que promueve el uso de herramientas tecnológicas en dichos programas y que procura siempre enfoques multidisciplinarios o interdisciplinarios por medio de la implementación de módulos que son pensados para un estudiante que emerge de la heterogeneidad y puede responder de manera positiva a la educación a distancia, que es discriminada de la siguiente manera:

Sistema de educación abierta, educación virtual, universidad virtual, campus virtual, instrucción asistida por computadora, educación no presencial, educación telemática, educación semiabierta, extensión universitaria abierta, universidad del conocimiento virtual, biblioteca digital, biblioteca virtual, educación informal, educación semipresencial, videoconferencias, etc., todas ellas ubicadas en las modalidades no escolarizadas y mixtas descritas en la Ley General de Educación. (Cervantes, Herrera y Parra, 2011, pp. 158-159). 
Uno de los avances que se han tenido en la educación a distancia es el enfoque hacia la virtualidad como herramienta de apoyo en la utilización de campus virtuales, bibliotecas, todos ellos tomando provecho de las TIC. Cada universidad tiene normatividades internas que hacen que estas herramientas sean implementadas con base en sus necesidades.

Finalmente, otros enfoques que permean la educación superior son la internacionalización y la globalización. La primera se debe ver como la oportunidad de reivindicación del rol de la educación superior en México, rescatándola de factores como la globalización y el neoliberalismo (Castellanos y Luna, 2014). Esta sería la manera en que los pueblos se preocuparían de las necesidades educativas contextuales de cada región. En cuanto a la globalización, que trae consigo el enfoque neoliberal a cuestas, se debería replantear hacia una "globalización de la solidaridad", que se ha estado trazando en la propuesta de integración ALBA (Alternativa Bolivariana para América Latina y el Caribe) -que lucha contra pobreza y la exclusión social- como una oposición al neoliberalismo. (Castellanos y Luna, 2014). Estas antítesis de internacionalización y globalización serían buenas estrategias para contrarrestar los efectos nocivos que actualmente estas aportan a la educación superior, y así rescatarlas del neoliberalismo y su mercantilización educativa.

\subsection{Tendencias de educación superior en México}

Las tendencias en cuanto a educación superior en México responden a los modelos socioeconómicos neoliberales y de globalización que se vienen presentando en la región desde la década de los setenta. Es por esta razón que las tendencias que se dan para atender a estos modelos se direccionan hacia la inclusión, la calidad educativa, la oferta de programas en entidades públicas y privadas y facilidades de acceso.

\section{Tendencia hacia la inclusión}

Apunta hacia la inclusión de los pueblos aborígenes con los que cuenta México, para darles la oportunidad de ingresar al sistema educativo, incluyendo el nivel superior. Lo malo es que las condiciones de bajos recursos económicos y las distancias que afrontan estas poblaciones hacen que no puedan acceder a este nivel de formación profesional o permanecer en él (Bastida, 2012).

\section{Tendencia a la calidad educativa}

Atañe en especial al gobierno para regularla en el sector público. Las instituciones de carácter privado pueden vincular sus programas de estudio al RVOE (Sistema de Reconocimiento de Validez Oficial de Estudios). Para la SEP (Secretaría de Educación Pública de México), la calidad de los programas en las instituciones de educación superior 
privadas y públicas se logra cuando entidades como el Consejo para la Acreditación de la Educación Superior A. C. (COPAES) o los Comités Interinstitucionales para la Evaluación de la Educación Superior (CIIES) se ocupan de hacer intervenciones de evaluaciones externas en las instituciones (De Garay, 2013). De esta manera, los procesos de calidad educativa tienden a volverse obligatorios debido a su proliferación y se convierten en una nueva estrategia mercantil que puede justificar sus costos por contar con este tipo de certificaciones. De este modo, se puede ver que la calidad educativa es algo que es independiente de cada institución.

\section{Tendencia a una oferta de programas}

Implica una amplia oferta educativa en todos sus niveles: técnico, tecnológico, profesional asociado, licenciatura y posgrado. Lo que no es suficiente es el tema de la cobertura, a pesar de que cuenta con la educación a distancia y abierta con enfoques virtuales para ofrecer estos programas como opción de acceso.

\subsection{Modelos de educación superior en México}

Los modelos hallados en este rastreo documental de México entre 2005 y 2013 fueron redistribuidos en cuatro grandes categorías:

\section{Modelos normativos}

Estos se refieren a la normatividad (leyes y políticas públicas) que se tienen en México para afrontar el tema de la educación intercultural, por ejemplo, el Plan Sectorial de Educación, que es avalado por la Secretaría de Educación Pública en su objetivo II, que se direcciona a eliminar las barreras que existen en el modelo convencional de educación superior y a involucrar a los pueblos originarios para que exista un intercambio de saberes por medio de una pedagogía intercultural y una equidad entre las culturas. $\mathrm{Al}$ respecto, Bastida (2012) expone que "la universidad es un espacio ideal para promover diversas perspectivas epistemológicas, impulsar distintas formas de construir y acceder al conocimiento, y coadyuvar en el logro de una vida buena para todos" (p. 374). De esta manera, los objetivos de la universidad son crear lazos de igualdad y de respeto por la diversidad, promover el enriquecimiento bilateral de las culturas que intervienen y fomentar el respeto hacia las lenguas originarias. Esta es una estrategia que además se desarrolla para la preservación de la cultura de los pueblos originarios y una lucha por contribuir a la cohesión social.

\section{Modelos de inclusión educativa}

Estos surgen debido a la necesidad que tiene México, ya que cuenta con población originaria, la cual constitucionalmente también tiene derecho a la educación superior. Entidades como la UNESCO se aúnan diciendo que es así como se debe propender 
hacia la eliminación de la exclusión social dentro del sistema educativo (Alcántara y Navarrete, 2014, p. 214). Incluye además a otros grupos sociales, como el afromexicano y personas en condición de discapacidad, entre otros, que son importantes y han de tenerse en cuenta. El reto es que por medio de la inclusión educativa se pueda promover la cohesión social en el pueblo mexicano.

\section{Modelos políticos}

Estos atienden a las políticas internacionales que se emiten a los gobiernos, los cuales son forzados a cumplir con unos mínimos reguladores para poder seguir subsistiendo y estar en sintonía con los demás países. El neoliberalismo es uno de los fenómenos que más ha tenido influencia en la educación superior, ya que se debe reestructurar todo el marco educativo en torno a él. La educación superior mexicana no ha sido la excepción y tuvo un choque neoliberal cuando debió apuntar su oferta educativa hacia la privatización y hacia la apertura de instituciones que cualifican la mano de obra, la cual es necesaria debido a requerimientos de calidad en el sector productivo, por ejemplo, en procesos de TLC con los países vecinos: Canadá y U. S. A. (De Garay, 2013), que dentro de sus condiciones exigen calidad y ven en la educación terciaria a nivel técnico una buena estrategia para cumplir con estos requerimientos.

\section{Modelos educativos}

Estos están diseñados para cumplir con los requerimientos sociopolíticos que se dan a la educación superior, la cual intenta responder a tales requerimientos por medio de la educación técnica, a distancia y abierta, permeadas por enfoques virtuales. A pesar de que en México no hay una reglamentación adecuada para estos modelos, se cuenta con el apoyo de instituciones como la SEP (Secretaría de Educación Pública) -que brinda apoyo a la educación abierta y a distancia-, la ANUIES (Asociación Nacional de Universidades e Instituciones de Educación Superior) - que apoya a las universidades e instituciones de educación superior- y, finalmente, el SINED (Sistema Nacional de Educación a Distancia) - que soporta a la educación a distancia en la inclusión de procesos de tecnología y virtualización-(Cervantes, Herrera y Parra, 2011).

\section{A manera de conclusión}

Este panorama de la educación superior en Argentina, Chile y México evidencia la inserción de los modelos neoliberales y de globalización que se han apoderado del sentido inicial de cientificidad de la educación. Algo positivo que se puede rescatar es que países como Argentina y México cuentan con tendencias y enfoques dirigidos hacia la igualdad social, la inclusión, la diversidad cultural y la interculturalidad de sus pueblos 
originarios; especialmente en Argentina, existe un interés por las comunidades afroamericanas. Caso contrario ocurre en Chile, ya que las minorías (nativos y afroamericanos) no cuentan con un aval normativo para su reivindicación, siendo los primeros mejor librados que los segundos.

Lamentablemente, la privatización es una de las secuelas neoliberales que ha golpeado a la educación superior y que ha originado desigualdad social al excluir a los jóvenes de bajos recursos, quienes deben recurrir a préstamos de larga duración e intereses para poder acceder a ella. Otro producto del modelo neoliberal es la calidad educativa, que constriñe a la educación superior de estos países latinoamericanos a adoptar requerimientos de cualificación en sus procesos de manera tal que sirvan para cualificar el conocimiento, entorpeciendo así el sentido inicial de la universidad, que es el de generar ciencia y sujetos con pensamiento crítico y no sujetos que se cualifican por medio de carreras técnicas y tecnológicas de corta duración para dar respuesta a las necesidades del mercado.

En cuanto al modelo de globalización, este direcciona la educación superior en estos países al uso de la virtualidad mediada por las TIC para ofrecer una cobertura más eficaz, como es el caso de Argentina y México. Sin embargo, en Chile, a pesar de contar con esta herramienta, los programas no poseen una normatividad, lo cual genera así poco interés hacia ellos.

\section{Referencias}

Alcántara, A. y Navarrete, Z. (2014). Inclusión, equidad y cohesión social en las políticas de educación superior en México. Revista Mexicana de Investigación Educativa, 19(60), 213-239. Recuperado de goo.gl/xgHIbl

Bastida, M. (2012). Políticas públicas de las instituciones y programas interculturales de educación superior en México. En D. Mato (Coord.), Educación superior y pueblos indígenas y afrodescendientes en América Latina. Normas, políticas y prácticas (pp. 347-379). Caracas: IESALC-UNESCO.

Bernasconi, A. y Rojas, F. (2004). Informe sobre educación superior en Chile: 1980-2003. Santiago de Chile: UNESCO.

Brunner, J. J. y Uribe, D. (2007). Mercados universitarios: el nuevo escenario de la educación superior. Santiago de Chile: Ediciones Universidad Diego Portales. Recuperado de goo. $\mathrm{gl} / \mathrm{jQuWHD}$ 
Castellanos, S. y Luna, C. (2014). La internacionalización y la globalización neoliberal en el contexto de la educación superior en México. Revista Iberoamericana de Educación, 49(7). Recuperado de http://rieoei.org/2776.htm

Catani, A., Campbell, J. y De Sousa, R. (2005). La educación superior en Chile: continuidades y desafios. Fundamentos en Humanidades, 6(11), 9-20. Recuperado de goo. $\mathrm{gl} / 3 \mathrm{xTV} 4 \mathrm{G}$

Cervantes, F., Herrera, A. y Parra, P., (2011). Leyes, normas y reglamentos que regulan la educación superior a distancia y en línea en México. En A. Camacho (Ed.), Leyes, normas y reglamentos que regulan la educación superior a distancia y en línea en América Latina y el Caribe (1.a ed.) (pp. 143-164). Ecuador: Universidad Técnica Particular de Loja.

Congreso de Argentina. (30 de septiembre de 1985). Ley n.o 23.302. Por medio de la cual se crea la Comisión Nacional de Asuntos Indígenas. Boletín Oficial n.o 25.803 del 12 de noviembre de 1985. Recuperado de goo.gl/oecEV0

. (29 de abril de 1993). Ley n.o 24.195 Federal de Educación. Boletín Oficial n.o 27.632 del 5 de mayo de 1993. Recuperada de goo.gl/RzugO8

. (20 de julio de 1995). Ley n.o 24.521. Ley Nacional de Educación Superior. Boletín Oficial n.o 28.204 del 10 de agosto de 1995. Recuperada de goo.gl/prwjw T

. (7 de septiembre de 2005). Ley n.o 26.058. Ley de Educación Técnico Profesional. Boletín Oficial n.o 30.735 del 9 de septiembre de 2005. Recuperado de goo.gl/dgzYQ4

. (14 de diciembre de 2006). Ley n.o 26.206 de 2006. Ley de Educación Nacional. Boletín Oficial n.o 31.062 del 28 de diciembre de 2006. Recuperado de goo.gl/m8WFOW

Congreso de los Estados Unidos Mexicanos. (México). (9 de julio de 1993). Ley General de Educación. Diario Oficial de la Federación (separata) del 13 de julio de 1993. Recuperado de goo.gl/PgXzeW . (29 de diciembre de 1978). Ley para la Coordinación de la Educación Superior. Diario Oficial de la Federación del 29 de diciembre de 1978. Recuperado de goo. gl/0gThui

De Garay, A. (2013). La expansión y diversificación de la educación superior privada en México en los primeros diez años del siglo XXI. Espacio Abierto. Cuaderno Venezolano de Sociología, 22(3), 413-436. Recuperado de goo.gl/fXZngE 
Escobar, M. R. Et al. (2004). Estado del arte del conocimiento producido sobre jóvenes en Colombia 1985-2003. Bogotá: Programa Presidencial Colombia-Joven, GTZ. - UNICEF, Universidad Central.

Farcas, D. (2010). Educación a distancia: experiencia del e-learning en Chile. En C. Rama y J. Pardo (Eds.), La educación superior a distancia: miradas diversas desde Iberoamérica (pp. 64-75). España: VirtualEduca-Inteved. Recuperado de goo.gl/GYODlw

Ministerio de Educación de Chile. (10 de marzo de 1990). Ley Orgánica Constitucional de Enseñanza n.o 18.962. Recuperado de goo.gl/hG9W4V

Martínez, J. (2011). La universidad productora de productores: entre biopolítica y subjetividad. Bogotá: Ediciones Unisalle.

Millán, M. (2012). Educación superior y pueblos originarios y afrodescendientes en Argentina. En D. Mato (Coord.), Educación superior y pueblos indígenas y afrodescendientes en América Latina. Normas, políticas y prácticas (pp. 103-138). Caracas: IESALC-UNESCO.

Ministerio de Cultura y Educación (Argentina). (11 de julio de 1997). Resolución n.o 1168/97. Educación Superior. Carreras de posgrado - Estándares y criterios. Boletín Oficial n.o 28.735 del 22 de septiembre de 1997. Recuperado de goo.gl/MeOJd5

Ministerio de Educación (Chile). (10 de marzo de 1990). Ley n. ${ }^{\circ}$ 18.962, Orgánica Constitucional de Enseñanza (LOCE). Diario Oficial de la República de Chile n.o 33.617 del 10 de marzo de 1990. Recuperado de goo.gl/eyxjne

Ministerio de Educación, Ciencia y Tecnología (Argentina). (29 de diciembre de 2004). Resolución n.o 1717/2004. Educación a Distancia. Boletín Oficial n.o 30.568 del 12 de enero de 2005. Recuperado de goo.gl/Q85ykM

Mollis, M. (2008). Las reformas de la educación superior en Argentina para el nuevo milenio. Avaliação: Revista da Avaliação da Educação Superior, 32(6), 509-532. DOI: 10.1590/ S1414-40772008000200012

Mora, M. (2012). Educación superior, interculturalidad y legislación en Chile. En D. Mato (Coord.), Educación superior y pueblos indígenas y afrodescendientes en América Latina. Normas, políticas y prácticas (pp. 211-244). Caracas: IESALC-UNESCO.

Ottone, E. (Dir.) y Sojo, A. (Coord.). (2007). Cohesión social: inclusión y sentido de pertenencia en América Latina y el Caribe. Santiago de Chile: Naciones Unidas. Recuperado de goo. $\mathrm{gl} / \mathrm{t} 6 \mathrm{oFvZ}$ 
ISSN: 0124-3551 / Año 18, No 28 / enero-junio / pp. 43-70

Poder Ejecutivo Nacional de Argentina. (22 de enero de 1998). Decreto Reglamentario 81/1998. Educación Superior. Educación a Distancia. Boletín Oficial n.o 28.823 del 27 de enero de 1998. Recuperado de goo.gl/gX5J5F

- (2 de octubre de 2001). Decreto Reglamentario n.o 1232/2001. Educación Superior. Sistema Integrado - Normas. Boletín Oficial n.o 29.747 del 5 de octubre de 2001. Recuperado de goo.gl/iyvqQN

Rojas, M. L. (2012). Educación superior en Argentina: ¿un sistema fuera de control? Revista de la Educación Superior, 41(161), 93-114. Recuperado de goo.gl/y42xpV

Santander, W., Schalk A., Zavando S. y Durán J. (2011). Leyes, normas y reglamentos que regulan la educación superior a distancia y en línea en Chile. En A. Camacho (Ed.). Leyes, normas y reglamentos que regulan la educación superior a distancia y en línea en América Latina y el Caribe (1.a ed.) (pp. 67-82). Ecuador: Universidad Técnica Particular de Loja.

Santángelo, H. (2011). Leyes, normas y reglamentos que regulan la educación superior a distancia y en línea en Argentina. En A. Camacho (Ed.). Leyes, normas y reglamentos que regulan la educación superior a distancia y en línea en América Latina y el Caribe (1.a ed.) (pp. 11-24). Ecuador: Universidad Técnica Particular de Loja.

Subsecretaría de Educación Superior. (2013a). Subsecretaría de Educación Superior. Recuperado de http://www.ses.sep.gob.mx/direc_coordi.html

Subsecretaría de Educación Superior. (2013b). Subsecretaría de Educación Superior. Recuperado de http://www.ses.sep.gob.mx/publicas_federales.html

Subsecretaría de Educación Superior. (2013c). Subsecretaría de Educación Superior. Recuperado de http://www.ses.sep.gob.mx/institutos_tecnologicos.html

Vejar, J. (2013). Reflexiones en torno a mercantilización de la universidad y sus desacoples con el mercado laboral. Tensiones de un modelo neoliberal de profesionalización en Chile. Espacio Abierto. Cuaderno Venezolano de Sociología, 22(1), 71-98. Recuperado de goo.gl/5UVoVI

Weller, J. (2011). Panorama de las condiciones de trabajo en América Latina. Revista Nueva Sociedad, 232, 32-49. Recuperado de goo.gl/mthSa4 